\title{
Influence of stimuli color on steady-state visual evoked potentials based BCI wheelchair control
}

\author{
Rajesh Singla ${ }^{1}$, Arun Khosla ${ }^{2}$, Rameshwar $\mathrm{Jha}^{3}$ \\ ${ }^{1}$ Department of Instrumentation and Control Engineering, Dr BR Ambedkar National Institute of Technology, Jalandhar, Punjab, India \\ ${ }^{2}$ Department of Electronics and Communication Engineering, Dr. B. R. Ambedkhar National Institute of Technology Jalandhar, \\ Punjab, India \\ ${ }^{3}$ Director General, IET Bhaddal, Ropar, Punjab, India \\ Email: rksingla1975@gmail.com, arun.khosla@gmail.com, rjharjha@yahoo.com
}

Received 21 August 2013; revised 25 September 2013; accepted 8 October 2013

Copyright (C) 2013 Rajesh Singla et al. This is an open access article distributed under the Creative Commons Attribution License, which permits unrestricted use, distribution, and reproduction in any medium, provided the original work is properly cited.

\begin{abstract}
In recent years, Brain Computer Interface (BCI) systems based on Steady-State Visual Evoked Potential (SSVEP) have received much attention. This study tries to develop a SSVEP based BCI system that can control a wheelchair prototype in five different positions including stop position. In this study four different flickering frequencies in low frequency region were used to elicit the SSVEPs and were displayed on a Liquid Crystal Display (LCD) monitor using LabVIEW. Four stimuli colors, green, red, blue and violet were used to investigate the color influence in SSVEPs. The Electroencephalogram (EEG) signals recorded from the occipital region were segmented into 1 second window and features were extracted by using Fast Fourier Transform (FFT). One-Against-All (OAA), a popular strategy for multiclass SVM, is used to classify SSVEP signals. During stimuli color comparison SSVEP with violet color showed higher accuracy than that with green, red and blue stimuli.
\end{abstract}

Keywords: Steady-State Visual Evoked Potential; Brain Computer Interface; Support Vector Machines

\section{INTRODUCTION}

The Brain Computer Interface (BCI) system provides a direct communication channel between human brain and the computer without using brain's normal output pathways of peripheral nerves and muscles [1,2]. By acquiring and translating the brain signals that are modified according to the intentions, a BCI system can provide an alternative, augmentative communication and control options for individuals with severe neuromuscular disorders, such as spinal cord injury, brain stem stroke and Amyo- trophic Lateral Sclerosis (ALS).

Electroencephalography (EEG) is a non-invasive way of acquiring brain signals from the surface of human scalp, which is widely accepted due to its simple and safe approach. The brain activities commonly utilized by EEG based BCI systems include Event Related Potentials (ERPs), Slow Cortical Potentials (SCPs), P300 potentials, Steady-State Visual Evoked Potentials (SSVEPs) etc. Among them SSVEPs are attracted due to its advantages of requiring less or no training, high Information Transfer Rate (ITR) and ease of use [1,3].

SSVEPs are oscillatory electrical potentials that are elicited in the brain when the person is visually focusing his/ her attention on a stimulus that is flickering at frequency $6 \mathrm{~Hz}$ or above [4]. These signals are strong in occipital region of the brain and are nearly sinusoidal waveform having the same fundamental frequency as the stimulus and including some of its harmonics. By matching the fundamental frequency of the SSVEP to one of the stimulus frequencies presented, it is possible to detect the target selected by the user. Considering the amplitudes of SSVEPs induced, the stimuli frequencies are categorized into three ranges, centred at $15 \mathrm{~Hz}$ low frequency, $31 \mathrm{~Hz}$ medium frequency and $41 \mathrm{~Hz}$ high frequency respectively [5].

There are many research groups that are designing SSVEP based BCI systems. Lalor et al. [6] developed the control for an immersive 3D game using SSVEP signal. Muller and Pfurtscheller [7] used SSVEPs as the control mechanism for two-axis electrical hand prosthesis. Recently, Lee et al. [8] presented a BCI system based on SSVEP to control a small robotic car.

One of the main considerations during the development of a BCI system is to improve the classifiers accuracy, as that can affect the overall system accuracy and thus the ITR. In this research work the Support Vector 
Machine (SVM) method was carried out for the classification of a multiclass SSVEP signal.

The retina of human eye contains rod and cone cells. The rod cells detect the amount of light and cone cells distinguish the color. There are three kinds of cone cells and are conventionally labeled as Short (S), Medium (M), and Long (L) cones according to the wavelengths of the peaks of their spectral sensitivities. S, M and L cone cells are therefore sensitive to blue (short-wavelength), green (medium-wavelength) and red (long-wavelength) light respectively. The brain combines the information from each cone cells to give different perceptions to different colors; as a result, the SSVEP strength elicited with different colors of the stimuli will be different.

\section{MATERIALS AND METHODS}

\subsection{System Configuration}

Figure 1 illustrates the block diagram of the proposed SSVEP based wheelchair control system, which includes visual stimuli developed using LabVIEW and displayed on a Liquid Crystal Display (LCD) monitor, EEG acquisition unit, signal processing unit with feature extraction and classification algorithms, hardware interface and a wheelchair prototype.

\subsection{Subject}

Ten right handed healthy subjects (seven males and three females, aged 22 - 27 years), with normal or corrected to normal vision participated in the experiment. All of them had normal color vision and not had any previous BCI experience. Prior starting, subjects were informed about the experimental procedure and required to sign a consent form.

\subsection{Stimuli}

The RVS for eliciting SSVEP responses can be presented

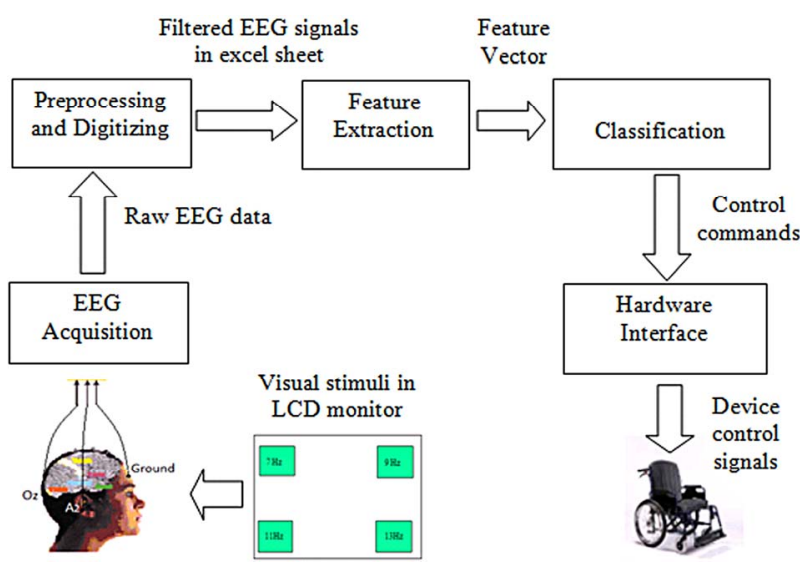

Figure 1. Conceptual block diagram of the proposed SSVEP based wheelchair control system. on a set of Light Emitting Diodes (LEDs) or on a Liquid Crystal Display (LCD) monitor [9]. In this study RVS displayed using LCD monitor due to the flexibility in changing the color of flickering bars, and were designed using LabVIEW software (National Instrument Inc., USA). Four colors: green, red, blue and violet were included in the experiment. Background color selected as black. Four frequencies 7, 9, 11 and $13 \mathrm{~Hz}$, in the low frequency range were selected, as the refreshing rate of LCD monitor is $60 \mathrm{~Hz}[10]$ and the high amplitude SSVEPs are obtained at lower frequencies [5]. The visual stimuli were square $(4 \mathrm{~cm} \times 4 \mathrm{~cm})$ in shape and were placed on four corners of the LCD screen.

\subsection{Experimental Setup}

The subjects were seated $60 \mathrm{~cm}$ in front of the visual stimulator as shown in Figure 2. EEG signals were recorded using RMS EEG-32 Super Spec system (Recorders and Medicare System, India). The SSVEP potential recorded from occipital region using $\mathrm{Ag} / \mathrm{AgCl}$ electrodes were amplified and connected to the adaptor box through head box. Adaptor box consist the circuitry for signal conditioning and further connected to the computer via USB port. This system can record 32 channels of EEG data. The electrodes were placed as per the international 10 - 20 system. The skin-electrode impedance was maintained below $5 \mathrm{~K} \Omega$. The EEG signals were filtered by using a $3-50 \mathrm{~Hz}$ band pass filter and a $50 \mathrm{~Hz}$ notch filter. Signals were sampled at $256 \mathrm{~Hz}$ and the sensitivity of the system was selected as $7.5 \mu \mathrm{V} / \mathrm{mm}$.

In training session the electrodes were placed at the $\mathrm{O}_{1}$, $\mathrm{O}_{2}$ and $\mathrm{O}_{\mathrm{z}}$ regions of the scalp. The reference electrodes were placed on the right and left earlobes $\left(A_{1}\right.$ and $\left.A_{2}\right)$ and ground electrode on $\mathrm{F}_{\mathrm{pz}}$. First collected the SSVEP data for all the four frequencies with green color and then repeated the experiment for red and violet colors in another session. Subject 2 performed with blue color

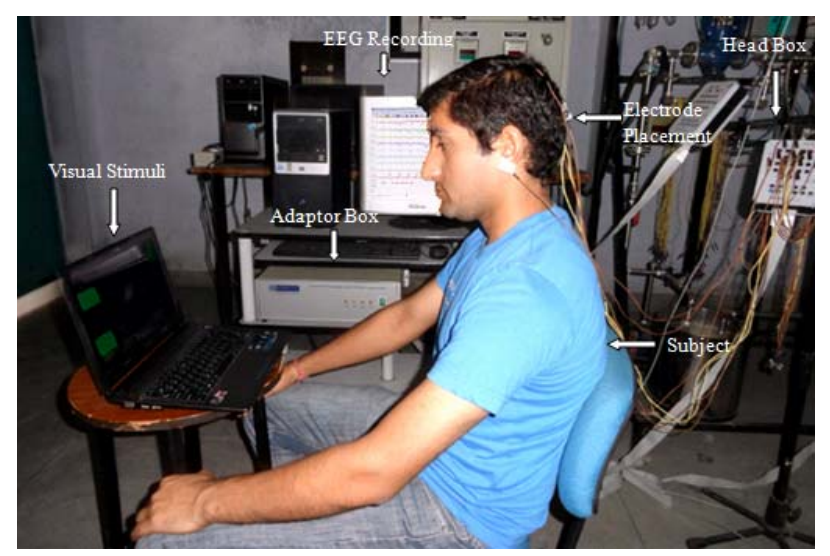

Figure 2. Experimental set up for SSVEP data acquisition (Courtesy-Department of Instrumentation and Control Engineering, National Institute of Technology, Jalandhar). 
stimuli in order to analyze the effect of blue stimuli on SSVEP. The interval between the sessions was 10 minutes. Initially the subjects were required to close their eyes for recording 2 minutes of baseline signal and then given 5 minutes to adapt to the flickering stimulus placed in front of them.

During experiments, the subjects were directed to focus on a particular frequency for 5 second duration followed by 5 second rest period. During focusing, the subjects were instructed to avoid eye movements or blinking. The event markers were used to indicate the starting and ending time of each frequency. In a single trial, each of the four frequencies was performed three times and the same procedure was repeated for another three trials. 5 minutes break was given in between each trial. The time for completing one session was about 30 minutes.

\subsection{Feature Extraction}

The frequency features of SSVEPs can easily extracted by using Fast Fourier Transform (FFT) [11]. The EEG signals recorded from $\mathrm{O}_{\mathrm{z}}-\mathrm{A}_{2}$ channel were digitized and segmented into 1 second time window in every 0.25 seconds. MATLAB was used for developing the FFT program. Figure 3 shows the amplitude spectra of SSVEP induced by $7 \mathrm{~Hz}$ stimulation. The coefficients at the fundamental and second harmonics of all the four target frequencies obtained from the amplitude spectra were considered as the feature vector for classification.

\subsection{Classification}

The SVM technique introduced by Vapnik in [12] is basically a binary classifier which can discriminate between two classes by using an optimal hyperplane which maximize the margin between the two classes. Kernel functions provide a convenient method for mapping the data space into a high-dimension feature space without computing the non-linear transformation [13]. The common kernel functions are linear, quadratic, polynomial and radial basis function (rbf).

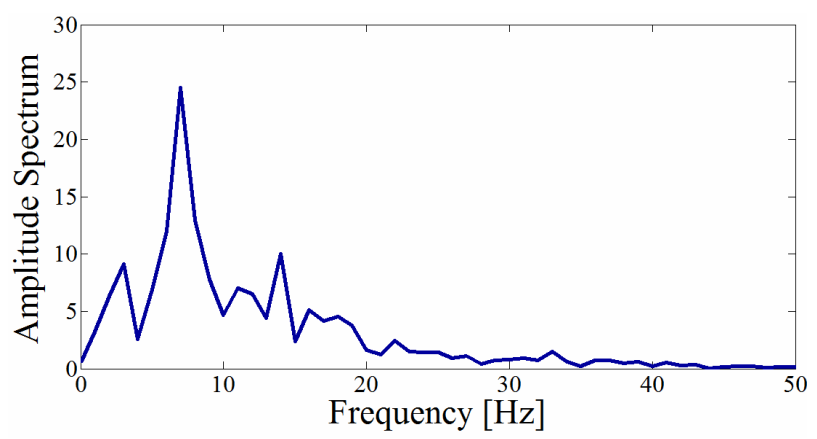

Figure 3. Amplitude spectra of SSVEP in response to $7 \mathrm{~Hz}$, recorded from $\mathrm{O}_{\mathrm{z}}-\mathrm{A}_{2}$ channel of subject 4 . First and second harmonics can be found clearly.
SVM training and classification was done by using MATLAB Bioinformatics toolbox. One-Against-All (OAA) method [12] was adopted for getting a multiclass SVM. The formulation of this mode states that a data point would be classified under a certain class if that class's SVM accepted it while rejected by all other classes SVMs. In this mode four binary SVMs were trained, each for one of the four frequencies. After training, there develop a structure having the details of the SVM like the number of support vectors, alpha, bias etc.

\subsection{Hardware and Implementation}

The wheelchair prototype is shown in Figure 4. Motor driver IC (IC L293D) was used to control two motors $\left(\mathrm{M}_{1}\right.$ and $\left.\mathrm{M}_{2}\right)$ of the wheelchair. By changing the polarity of the signal given to the motors, through the motor driver IC, it is possible to move the motors in both forward and backward directions.

The parallel port of the computer was used to send out eight data bits. The first four data pins i.e. $\mathrm{D}_{0}, \mathrm{D}_{1}, \mathrm{D}_{2}$, and $\mathrm{D}_{3}$ were used to interface the control signal to the motor IC. Positive and negative of the right motor was given through $\mathrm{D}_{0}$ and $\mathrm{D}_{1}$ and that of left motor was by using $\mathrm{D}_{2}$ and $\mathrm{D}_{3}$. Rest of the data pins was not used. Interfacing program was developed using MATLAB.

The control commands used to change the polarity of the motors for each movement of the wheelchair were presented in Table 1. Forward movement of both right

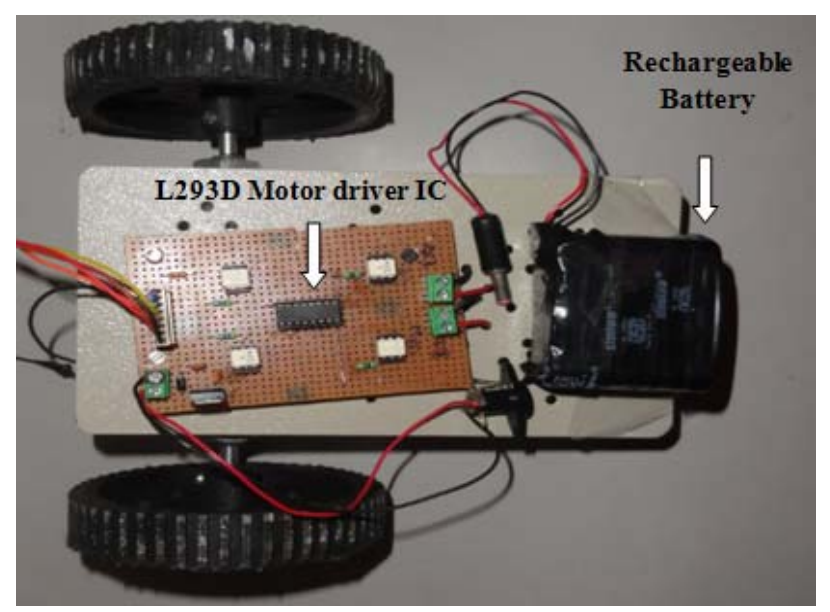

Figure 4. Wheelchair prototype for SSVEP based BCI control.

Table 1. Control logic for wheelchair movements.

\begin{tabular}{ccccc}
\hline \multicolumn{2}{c}{ Right Motor $\left(\mathbf{M}_{\mathbf{1}}\right)$} & \multicolumn{2}{c}{ Left Motor $\left(\mathbf{M}_{\mathbf{2}}\right)$} & Movement Direction \\
\cline { 1 - 4 }+ & - & + & - & \\
\hline 1 & 0 & 1 & 0 & Forward (F) \\
0 & 1 & 0 & 1 & Backward (B) \\
0 & 0 & 1 & 0 & Right (R) \\
1 & 0 & 0 & 0 & Left (L) \\
0 & 0 & 0 & 0 & Stop \\
\hline
\end{tabular}


$\left(\mathrm{M}_{1}\right)$ and left $\left(\mathrm{M}_{2}\right)$ motor results in the forward direction motion. Left motor forward and stop position of right motor will provide right movement of the wheelchair. Left motor stopped and a forward movement of right motor results left rotation of the wheelchair. The backward movement of both motor together provides the device to move backward. The stop positions of both the motor together results in the stopping of wheelchair.

The classifier outputs for each of the four frequencies and relax state were assigned to the five different movements of the wheelchair. For $7 \mathrm{~Hz}$ detection, the output of the parallel port is $\left[\begin{array}{llll}1 & 0 & 1 & 0\end{array}\right]$ and will move the wheelchair in forward direction. $9 \mathrm{~Hz}$ would give $\left[\begin{array}{llll}0 & 0 & 1 & 0\end{array}\right]$ and will cause a right movement. $11 \mathrm{~Hz}$ detection delivers an output of $\left[\begin{array}{llll}1 & 0 & 0 & 0\end{array}\right]$ and will result in the left movement of the wheelchair. For $13 \mathrm{~Hz}$ the parallel port output is [ $\begin{array}{ll}0 & 1\end{array}$ 0 1] which results in a backward movement of the wheelchair. The classifier result for the relax state of the user is $\left[\begin{array}{llll}0 & 0 & 0 & 0\end{array}\right]$ and it will stop the wheelchair.

\section{RESULTS AND DISCUSSIONS}

After the text edit has been completed, the paper is ready for the template. Duplicate the template file by using the Save As command, and use the naming convention prescribed by your journal for the name of your paper. In this newly created file, highlight all of the contents and import your prepared text file. You are now ready to style your paper.

\subsection{Classifier Testing Results}

The feature vector extracted using FFT were used for classification. There have two separate data sets each for two different stimuli colors. The training dataset for each color consist 150 samples (30 samples for each of the four frequencies and 30 for rest signal) from each subject data i.e. a total of 1500 samples in a complete set. The data were normalized in the range of $[-1,+1]$ Individual SVMs were trained with different kernel functions. The kernels with maximum accuracies were selected for OAASVM formulation. For $7 \mathrm{~Hz}$ the Polynomial kernel with order 3 had got an accuracy of $100 \%$ for violet color and for $9 \mathrm{~Hz}$ quadratic kernel provides an accuracy of $98.69 \%$ for the same. Higher accuracy for $11 \mathrm{~Hz}$ was provided by linear kernel and is $95.43 \%$ for violet color. For $13 \mathrm{~Hz}$ violet color got an accuracy of $100 \%$ by using linear kernel. The OAA-SVM designed with optimal kernels provides an overall accuracy of $98.53 \%$ for violet during training.

Figure 5 presents the regression plots during training of SSVEPs elicited by green, red and violet stimuli. The regression value for green is 0.94021 and that of red is 0.95162 . The violet got a regression value of 0.98532 . This proves the superior performance of violet stimuli over green and red for eliciting SSVEPs.

\subsection{Wheelchair Interface}

In testing session the subjects were directed to perform two different sequences i.e. path $\mathrm{A}$ and path $\mathrm{B}$, each one with 8 movements including stop command. Each of the sequence was performed three times, thus each subject performed a total of 48 movements. The shape of the paths is shown in Figure 6.

Table 2 presents the sequences required to complete the paths and corresponding frequencies. The SSVEP data recorded from Oz-A2 channel was filtered, digitized and segmented into one second window in every $0.25 \mathrm{sec}$ onds and transformed into frequency domain using FFT.

To reduce the number of wrong selections, a requirement of three continuous detection of the same target was set to produce the particular command to the wheelchair.

Accuracy of the system is measured with the accurate

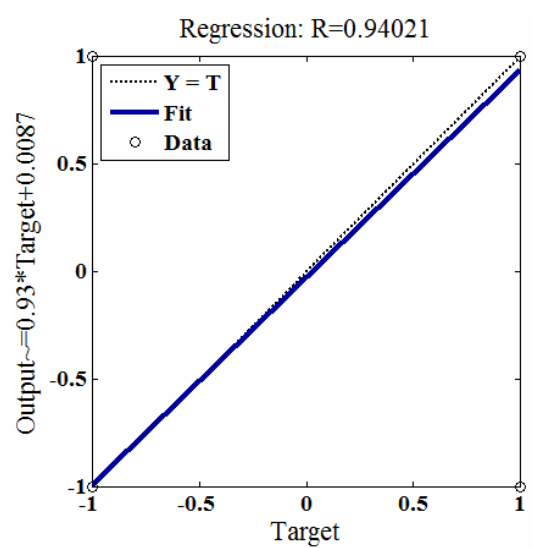

(a)

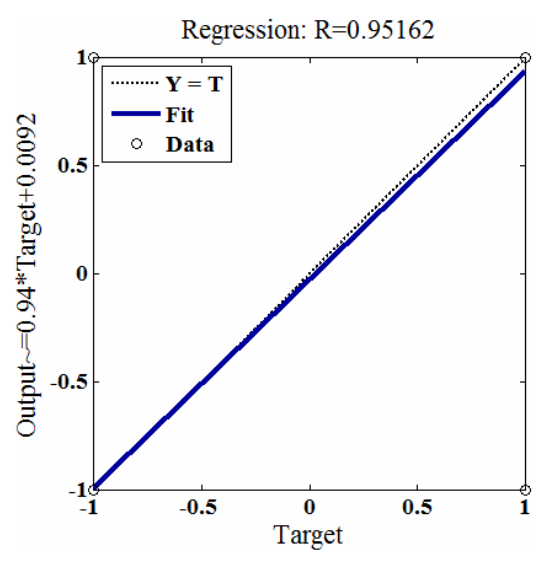

(b)

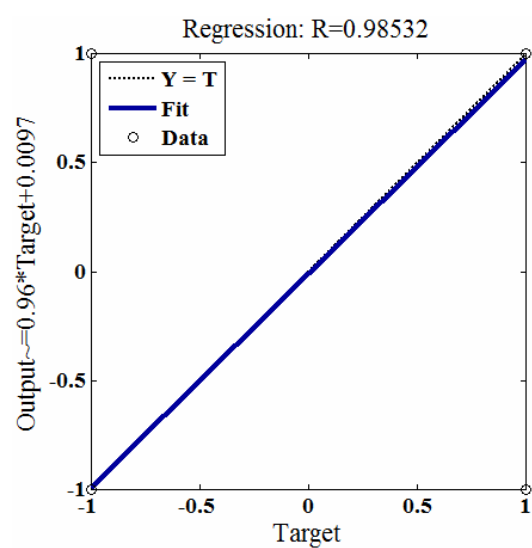

(c)

Figure 5. Comparison of regression plots for SSVEPs elicited by green, red and violet stimuli during classification of SSVEP data using OAA SVM. 
detections made by the subject out of the total number of the detections. The accuracy and the ITR of the system for 10 subjects with three stimuli colors using OAASVM classifier are presented in Table 3. Subject S4 got $100 \%$ accuracy by using OAA-SVM classifier with all the three stimuli colors. Result also indicates that violet color stimuli promise a SSVEP-BCI wheelchair control with good accuracy.

Wheelchair with violet stimuli achieves accuracy ranging from $79 \%-100 \%$ and in which nine subjects got accuracy higher than $89 \%$. Subject S9 showed comparatively poor performance and got accuracy $79.17 \%$ with violet stimuli.

The system was further experimented with OAA SVM classifier and blue stimuli for subject 2 and obtained 46 correct detections out of 48 total detections. The accu- racy is $95.83 \%$ and ITR is 23.86 bits/min respectively.

\section{CONCLUSIONS}

In this research OAA-SVM was constructed for SSVEP data classification. The motivation of this work is to improve the accuracy of SSVEP based BCI system by using optimal stimuli color. EEG signals were recorded by using RMS EEG-32 Super Spec system and SSVEP features extracted using FFT. SSVEPs were elicited using four different frequencies. Four different stimuli colors green, red, blue and violet were compared to get better performance. The amplitudes of first and second harmonics of SSVEP data were successfully used as the feature vector to train the classifier models. The result showed that SSVEPs with violet stimuli are better than that with
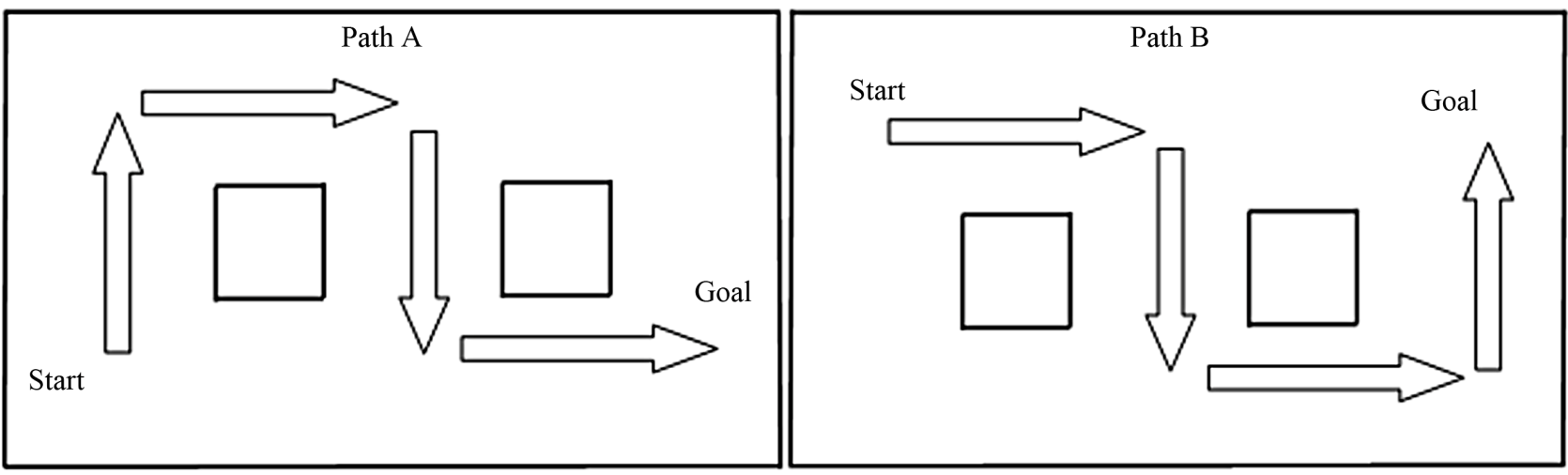

Figure 6. Shape of the paths used in testing session.

Table 2. Sequence of movements and corresponding frequencies.

\begin{tabular}{ccccccccccc}
\hline \multirow{2}{*}{ Path A } & Movement & F & R & F & R & F & L & F & Stop \\
& Frequency (Hz) & 7 & 9 & 7 & 9 & 7 & 11 & 7 & Relax \\
\hline \multirow{2}{*}{ Path B } & Movement & F & R & F & L & F & L & F & Stop \\
& Frequency (Hz) & 7 & 9 & 7 & 11 & 7 & 11 & 7 & Relax \\
\hline
\end{tabular}

Table 3. Number of correct detections (score), accuracy [\%] and itr [bits/min] for 10 subjects with green, red and violet stimuli using oaa-svm classifier.

\begin{tabular}{|c|c|c|c|c|c|c|c|c|c|}
\hline \multirow{2}{*}{ Subjects } & \multicolumn{3}{|c|}{ Green } & \multicolumn{3}{|c|}{ Red } & \multicolumn{3}{|c|}{ Violet } \\
\hline & Score & Acc. & ITR & Score & Acc. & ITR & Score & Acc. & ITR \\
\hline $\mathrm{S} 1 *$ & 46 & 95.83 & 23.86 & 46 & 95.83 & 23.86 & 47 & 97.92 & 25.61 \\
\hline $\mathrm{S} 2$ & 45 & 93.75 & 22.32 & 46 & 95.83 & 23.86 & 48 & 100 & 27.86 \\
\hline $\mathrm{S} 3$ & 40 & 83.33 & 16.06 & 41 & 85.42 & 17.17 & 43 & 89.58 & 19.58 \\
\hline $\mathrm{S} 4 *$ & 48 & 100 & 27.86 & 48 & 100 & 27.86 & 48 & 100 & 27.86 \\
\hline S5 & 44 & 91.67 & 20.9 & 44 & 91.67 & 20.9 & 46 & 95.83 & 23.86 \\
\hline S6 & 43 & 89.58 & 19.58 & 44 & 91.67 & 20.9 & 46 & 95.83 & 23.86 \\
\hline S7 & 42 & 87.5 & 18.34 & 36 & 75 & 12.13 & 43 & 89.58 & 19.58 \\
\hline S8 & 47 & 97.92 & 25.61 & 47 & 97.92 & 25.61 & 48 & 100 & 27.86 \\
\hline S9 & 34 & 70.83 & 10.41 & 35 & 72.92 & 11.25 & 38 & 79.17 & 14.01 \\
\hline $\mathrm{S} 10^{*}$ & 45 & 93.75 & 22.32 & 46 & 95.83 & 23.86 & 46 & 95.83 & 23.86 \\
\hline Average & 43.4 & 90.42 & 20.73 & 43.3 & 90.21 & 20.74 & 45.3 & 94.38 & 23.39 \\
\hline
\end{tabular}

*Female Participants. 
green, red and blue stimuli.

The future work may include the development of a SSVEP based BCI application system that can provide higher accuracy by using violet color as the stimuli color.

\section{ACKNOWLEDGEMENTS}

The author would like to thank the subjects who participated in the EEG recording session.

\section{REFERENCES}

[1] Wolpaw, J.R., Birbaumer, N., Heetderks, W.J., McFarland, D.J., Peckham, P.H., Schalk, G., Donchin, E., Quatrano, L.A., Robinson, C.J. and Vaughan, T.M. (2000) Brain computer interface technology: A review of the first international meeting. IEEE Transactions on Rehabilitation Engineering, 8, 164-173.

[2] Singla, R., Chambayil, B., Khosla, A. and Santosh, J. (2011) Comparison of SVM and ANN for classification of eye events in EEG. Journal of Biomedical Sciences and Engineering (JBISE), 4, 62-69.

[3] Berger, T.W., Chapin, J.K., Gerhardt, G.A., et al. (2007) International assessment of research and development in brain-computer interfaces: Report of the world technology evaluation center. Springer, Berlin.

[4] Cheng, M., Gao, X.R., Gao, S.K. and Xu, D. (2002) Design and implementation of a brain computer interface with high transfer rates. IEEE Transactions on Biomedical Engineering, 49, 1181-1186. http://dx.doi.org/10.1109/TBME.2002.803536

[5] Wang, Y.J., Wang, R.P., Gao, X.R., Hong, B. and Gao, S.K. (2006) A practical VEP-based brain-computer interface. IEEE Transactions on Neural Systems and Rehabilitation Engineering, 14, 234-240.

http://dx.doi.org/10.1109/TNSRE.2006.875576
[6] Lalor, E.C., Kelly, S.P., Finucane, C., Burke, R., Smith, R., Reilly, R.B. and McDarby, G. (2005) Steady-state VEP-based brain-computer interface control in an immersive 3D gaming environment. EURASIP Journal on Applied Signal Processing, 2005, 3156-3164. http://dx.doi.org/10.1155/ASP.2005.3156

[7] Muller-Putz, G.R. and Pfurtscheller, G. (2008) Control of an electrical prosthesis with an SSVEP-based BCI. IEEE Transactions on Biomedical Engineering, 55, 361-364. http://dx.doi.org/10.1109/TBME.2007.897815

[8] Lee, P.L., Chang, H.C., Hsieh, T.Y., et al. (2012) A brain wave actuated small robot car using ensemble empiricalmode decomposition based approach. IEEE Transactions on Systems, Man, and Cybernetics, Part A: Systems and Humans, 42, 1053-1064.

[9] Zhu, D.H., Bieger, J., Molina, G.G. and Aarts, R.M. (2010) A survey of stimulation methods used in SSVEP-based BCI system. Computational Intelligence and Neuroscience, 702357.

[10] Wang, Y., Wang, Y.-T. and Jung, T.-P. (2010) Visual stimulus design for high-rate SSVEP BCI. Electronics Letters, 46, 1057-1058. http://dx.doi.org/10.1049/el.2010.0923

[11] Muller-Putz, G.R., Scherer, R., Brauneis, C. and Pfurtscheller, G. (2005) Steady-state visual evoked potential (SSVEP)-based communication: Impact of harmonic frequency components. Journal of Neural Engineering, 2, 123-130. http://dx.doi.org/10.1088/1741-2560/2/4/008

[12] Vapnik, V. (1998) Statistical learning theory. John Wiley and Sons, Chichester.

[13] Song-Yun, X., Peng-Wei, W., Hai-Jun, Z. and Hai-Tao, Z. (2008) Research on the classification of brain function based on SVM. The 2nd International Conference on Bioinformatics and Biomedical Engineering, ICBBE 2008, 1931-1934. 